\title{
Non-uptake of viral load testing among people receiving HIV treatment in Gomba district, rural Uganda
}

Rita Nakalega ${ }^{1 *}$ D , Nelson Mukiza², George Kiwanuka ${ }^{3}$, Ronald Makanga-Kakumba ${ }^{4}$, Robert Menge ${ }^{5}$, Hajira Kataike ${ }^{1}$, Joel Maena', Carolyne Akello ${ }^{1}$, Patience Atuhaire ${ }^{1}$, Flavia Matovu-Kiweewa' ${ }^{1}$ Cynthia Ndikuno-Kuteesa ${ }^{3}$, Henry Debem ${ }^{6}$ and Andrew Mujugira ${ }^{3,7}$

\begin{abstract}
Background: Viral load (VL) testing is the gold-standard approach for monitoring human immunodeficiency virus (HIV) treatment success and virologic failure, but uptake is suboptimal in resource-limited and rural settings. We conducted a cross-sectional study of risk factors for non-uptake of VL testing in rural Uganda.

Methods: We conducted a cross-sectional analysis of uptake of $V L$ testing among randomly selected people with HIV (PWH) receiving anti-retroviral treatment (ART) for at least 6 months at all eight primary health centers in Gomba district, rural Uganda. Socio-demographic and clinical data were extracted from medical records for the period January to December 2017. VL testing was routinely performed 6 months after ART initiation and 12 months thereafter for PWH stable on ART. We used descriptive statistics and multivariable logistic regression to evaluate factors associated with non-uptake of VL testing (the primary outcome).

Results: Of 414 PWH, 60\% were female, and the median age was 40 years (interquartile range [IQR] 31-48). Most (62.3\%) had been on ART > 2 years, and the median duration of treatment was 34 months (IQR 14-55). Thirty three percent did not receive VL testing: $36 \%$ of women and $30 \%$ of men. Shorter duration of ART ( $\leq 2$ years) (adjusted odds ratio $[A O R] 2.38 ; 95 \% \mathrm{Cl}: 1.37-4.12 ; p=0.002$ ), younger age 16 -30 years (AOR 2.74; 95\% Cl:1.44-5.24; $p=0.002$ ) and $31-45$ years (AOR 1.92; $95 \% \mathrm{Cl} 1.12-3.27 ; p=0.017$ ), and receipt of ART at Health Center IV (AOR 2.85; 95\% Cl: 1.78-4.56; $p<0.001)$ were significantly associated with non-uptake of VL testing.

Conclusions: One-in-three PWH on ART missed VL testing in rural Uganda. Strategies to improve coverage of VL testing, such as VL focal persons to flag missed tests, patient education and demand creation for VL testing are needed, particularly for recent ART initiates and younger persons on treatment, in order to attain the third Joint United Nations Program on HIV/AIDS (UNAIDS) 95-95-95 target - virologic suppression for 95\% of PWH on ART.
\end{abstract}

Keywords: HIV, ART, Viral load, Testing, Uganda

\footnotetext{
* Correspondence: rnakalega@mujhu.org

${ }^{1}$ Makerere University-Johns Hopkins University (MU-JHU) Care LTD, Kampala, Uganda

Full list of author information is available at the end of the article
}

(c) The Author(s). 2020 Open Access This article is licensed under a Creative Commons Attribution 4.0 International License, which permits use, sharing, adaptation, distribution and reproduction in any medium or format, as long as you give appropriate credit to the original author(s) and the source, provide a link to the Creative Commons licence, and indicate if changes were made. The images or other third party material in this article are included in the article's Creative Commons licence, unless indicated otherwise in a credit line to the material. If material is not included in the article's Creative Commons licence and your intended use is not permitted by statutory regulation or exceeds the permitted use, you will need to obtain permission directly from the copyright holder. To view a copy of this licence, visit http://creativecommons.org/licenses/by/4.0/ The Creative Commons Public Domain Dedication waiver (http://creativecommons.org/publicdomain/zero/1.0/) applies to the data made available in this article, unless otherwise stated in a credit line to the data. 


\section{Introduction}

Eastern and Southern Africa is the epicenter of the global HIV epidemic, accounting for 800,000 (47\%) of the 1.7 million new HIV infections globally in 2018 [1, 2]. Between 2010 and 2018, the number of new HIV infections in Uganda decreased from 92,000 to 53,000 , a $43 \%$ reduction [1]. Despite these gains, Uganda is not on track to reach the UNAIDS 95-95-95 targets for HIV epidemic control i.e., 95\% of people with HIV (PWH) knowing their HIV status; $95 \%$ of people who know their status on treatment; and $95 \%$ of people on treatment with suppressed viral loads by 2030 [3]. In 2018, 84\% of PWH in Uganda knew their status, $72 \%$ were receiving ART and $64 \%$ of people on ART were virally suppressed [4]. Viral suppression, i.e., suppression of plasma viral load below the lower limit of detection for commercially available assays (HIV RNA $<50$ copies/ml), is the key indicator of HIV treatment success [5-7]. The World Health Organization (WHO) recommends the use of viral load (VL) testing as the gold standard to ensure viral suppression is achieved and sustained [8], but gaps in the VL cascade of care remain [9]. During JanuaryJune 2016, the proportion of people on ART ever receiving at least one VL test in seven sub-Saharan African countries was 9-91\% [2].

Uganda began scaling up HIV viral load monitoring in 2014, and the proportion of persons on ART receiving VL testing increased from $5 \%$ in 2014 to $50 \%$ in 2017 [2, 10]. However, nationally representative data on VL testing coverage are scarce among specific populations such as adolescents and young people who have lower prevalence of viral load suppression (42.5\%) than adults 5054 years $(74.2 \%)$ [11]. In one study of 397 adolescents aged 10-19 years in Uganda, only 238 (60\%) had ever received VL testing [11]. Factors limiting uptake of VL testing in resource-limited settings include lack of awareness among providers and patients of the benefits of VL testing, poor adherence to $\mathrm{WHO}$ and national guidelines on VL testing, overburdened health care workers, suboptimal training in quality assurance, weak health and laboratory systems, inefficient transport and result delivery systems, limited funding for the scale-up activities, and poor procurement processes [2, 12]. Factors associated with VL uptake in sub-Saharan Africa include older age, longer time on ART and one-stop HIV care models [13, 14].

Few studies have examined patient and health facilitylevel factors associated with non-uptake of VL testing in rural settings in sub-Saharan Africa [12, 13, 15]. Addressing these factors will inform programmatic implementation of WHO recommendations for universal VL testing, identify specific interventions to support VL monitoring, and help attainment of the third UNAIDS 95-95-95 target [3]. This study aimed to describe factors associated with non-uptake of VL testing among PWH in Gomba district, Uganda.

\section{Methods \\ Subjects and setting}

We conducted a cross-sectional study of PWH who received ART between January and December 2017 in Gomba district, rural Uganda. Gomba is one of 136 administrative districts in Uganda (population 160,075; 92\% rural; $49 \%$ female) [16] with eight primary health care centers providing comprehensive HIV services and ART to approximately 4200 PWH [17]. Uganda's healthcare system operates on a referral system, in which lower level facilities refer cases to the next level unit. Primary care facilities are organized by administrative division and include Health Center II (parish), Health Center III (sub-county) and Health Center IV (county). Gomba district had one Health Center IV (a mini hospital with surgical and obstetric services managed by a medical doctor) and 7 Health Center III facilities (each with a general outpatient clinic and maternity ward and led by clinical officers) [18]. Following the adoption of HIV 'test and treat' policies in 2016, all newly diagnosed PWH are immediately initiated on ART in accordance with Uganda treatment guidelines [19]. VL testing is performed 6 months after initiation of ART and 12 months thereafter for persons stable on ART [19]. Sociodemographic and clinical data are recorded on patient treatment cards (blue card) and PWH are asked to return to clinic every 3 months for adherence assessment and drug refills. At the scheduled visit for VL testing, the date of blood sample collection is documented on the client's blue card. Samples are sent from ART clinics to a district laboratory hub for transportation to the national reference laboratory. VL results are recorded in viral load registers and blue cards, and provided to PWH at subsequent clinic visits [17].

\section{Population and procedures}

Data were collected from all 8 primary care ART clinics during a 6-week period in mid-October and November 2018. We included all PWH on ART for at least 6 months. We excluded those with unknown duration on ART, including persons transferred-in from other centers. We used the Leslie Kish equation for crosssectional studies [20] to estimate the sample size of 414 participants, and the proportionate allocation method to determine the number of participants randomly selected from each health facility [21]. Each PWH was given a unique study identifier by facility staff and a list of codes was used to randomly select PWH from each facility. Data were extracted from paper and electronic ART registers and blue cards. The study team reviewed all extracted records to ensure data quality and perform 
corrections when data errors were identified. The ART start date was used to calculate the duration on ART at the time of the VL test. Dates of blood draw for VL testing, receipt of results at the clinic, and provision of results to PWH were recorded in a Microsoft $^{\circ}$ Excel database.

\section{Laboratory methods}

HIV testing is performed using serial rapid HIV-tests: Determine $^{\circ}$ HIV1/2 (Abbott, IL) [screening test], Stat$\mathrm{Pak}^{\ominus}$ HIV1/2 (Chembio Diagnostic Systems, Medford, NY) [confirmatory test], and SDBioline (Abbott, IL) [tie breaker test] according to national guidelines [22]. Plasma HIV RNA levels are quantified at the Central Public Health Laboratory using the COBASR TaqMan Analyzer or the COBASR AmpliPrep/COBAS TaqMan HIV-1 test, v2.0 (Roche Molecular Diagnostics, South Branchburg, NJ, USA) (17). Turnaround time for VL results is approximately $6-8$ weeks.

\section{Statistical analysis}

Non-uptake of VL testing (the primary outcome) was defined as having no VL test performed during the 12month study period. We used descriptive statistics and a multivariable logistic regression model to assess factors related to non-uptake of VL testing. Baseline factors evaluated included age, sex, marital status, ART duration, and health facility level. Backward elimination, starting with factors with $p \leq 0.2$ in bivariate analyses, was performed to select a final multivariate model. Goodness of fit was tested using the Hosmer-Lemeshow test [23]. Interaction and confounding were assessed during model building. Two-sided $p$-values of $\leq 0.05$ were considered statistically significant. Statistical analyses were performed using the Statistical Package for the Social Sciences (SPSS) version 20.0 (IBM Corp. Armonk, NY, USA).

\section{Results}

We enrolled a total of 414 PWH, of whom 250 (60\%) were female. The median age and duration on ART were 40 years (interquartile range [IQR] 31-48), and 34 months (IQR 14-55), respectively. One-in-three PWH (33\%) did not receive VL testing during the study period. Relative to older age ( $>46$ years), younger age (odds ratio [OR] 2.90; $p=0.001$ for $16-30$ years and OR $1.84 ; p=$ 0.02 for $31-45$ years) were significantly associated with non-uptake of VL testing uptake in univariate analysis (Table 1). Those on ART $\leq 2$ years (OR 2.24; 95\% $p=$ $0.002)$ were more likely to have VL testing non-uptake compared to those on ART $>4$ years. Similary, receipt of HIV care at Health Center IV level facility (OR 2.27; $p<$ 0.001 ) was also associated with VL testing non-uptake. We observed similar findings in the adjusted model. Younger age remained significantly associated with nonuptake of VL testing (adjusted odds ratio [AOR] 2.74;

Table 1 Participant characteristics and correlates of $V L$ testing non-uptake

\begin{tabular}{|c|c|c|c|c|c|c|}
\hline \multirow[b]{2}{*}{ Characteristic } & \multirow[t]{2}{*}{ Frequency (\%) } & \multirow[t]{2}{*}{ No VL test $(\%)$} & \multicolumn{2}{|c|}{ Univariate model } & \multicolumn{2}{|c|}{ Multivariable model } \\
\hline & & & OR $(95 \% \mathrm{Cl})$ & $p$-value & AOR (95\% Cl) & $p$-value \\
\hline \multicolumn{7}{|l|}{ Age (years) } \\
\hline $1-15$ & $24(5.8)$ & $6(25.0)$ & $1.13(0.41-3.11)$ & 0.81 & $1.37(0.48-3.93)$ & 0.55 \\
\hline $16-30$ & $76(18.4)$ & $35(46.1)$ & $2.90(1.58-5.33)$ & 0.001 & $2.74(1.44-5.24)$ & 0.002 \\
\hline $31-45$ & $182(44.4)$ & $64(35.2)$ & $1.84(1.11-3.07)$ & 0.018 & $1.92(1.12-3.27)$ & 0.017 \\
\hline$>46$ & $132(21.0)$ & $30(22.7)$ & Referent & & Referent & \\
\hline \multicolumn{7}{|l|}{ ART Duration } \\
\hline$\leq 2$ years & $156(37.7)$ & $67(42.9)$ & $2.24(1.35-3.71)$ & 0.002 & $2.38(1.37-4.12)$ & 0.002 \\
\hline$>2-4$ years & $127(30.7)$ & $35(27.6)$ & $1.31(0.65-1.97)$ & 0.67 & $1.35(0.75-2.44)$ & 0.32 \\
\hline$>4$ years & $131(31.6)$ & $33(25.2)$ & Referent & & Referent & \\
\hline \multicolumn{7}{|l|}{ Health Facility } \\
\hline Level III & $276(66.7)$ & $73(26.4)$ & Referent & & Referent & \\
\hline Level IV & $138(33.3)$ & $62(44.9)$ & $2.27(1.48-3.48)$ & $<0.001$ & $2.85(1.78-4.56)$ & $<0.001$ \\
\hline \multicolumn{7}{|l|}{ Gender } \\
\hline Male & $164(39.6)$ & $59(36.0)$ & Referent & & & \\
\hline Female & $250(60.4)$ & $76(30.4)$ & $0.77(0.51-1.18)$ & 0.24 & & \\
\hline \multicolumn{7}{|l|}{ Marital Status $^{\mathbf{a}}$} \\
\hline Married & $246(66.5)$ & $81(32.9)$ & Referent & & & \\
\hline Unmarried & $124(33.5)$ & $39(31.5)$ & $0.94(0.59-1.49)$ & 0.78 & & \\
\hline
\end{tabular}


95\% CI: $1.44-5.24 ; p=0.002$ and AOR 1.92; 95\% CI: $1.12-3.27 ; p=0.017$ ) for $16-30$ years and $31-45$ years, respectively. ART duration $\leq 2$ years (AOR 2.38; 95\% CI: $1.37-4.12 ; p=0.002)$ and receipt of ART at Health Center level IV (AOR 2.85; 95\% CI: 1.78-4.56; $p<0.001$ ) were significantly associated with VL non-uptake.

\section{Discussion}

In this cross-sectional study of 414 PWH receiving ART in rural Uganda, in which the median duration of HIV treatment was approximately 3 years, we found that onein-three PWH on ART did not receive a VL test during the 12-month study period. Younger age, shorter duration of ART, and receipt of HIV care at a Health Center IV facility were associated with non-uptake of VL testing.

Our finding that $33 \%$ of PWH on ART did not receive a VL test in 2017 is in agreement with prior work in South Africa in which 42, 32, and $26 \%$ that did not receive VL testing 6 months, 1 year and 2 years, respectively, after starting treatment [24]. Leakages in the VL cascade were observed in Mozambique where nonuptake of a first VL test was 60\% in 2015 [13] and in Cameroon where VL non-uptake was 76\% in 2017 [25]. Lack of VL testing limits the evaluation of treatment success, i.e., two consecutive VL measurements $<1000$ copies/ml [8], and assessment of HIV transmission risk to sexual partners and infants $[7,8]$. Prior to scale up of VL testing in Uganda in 2014, only 5\% of ART recipients had received VL testing; this proportion was $23 \%$ in $2015,22 \%$ in $2016,50 \%$ in 2017, 63\% in 2018 and $85 \%$ in 2019 [2, 10, 26, 27]. This finding of relatively high non-uptake of VL testing emphasizes the need for governments in resource-limited settings to scale up VL testing in support of "test and treat all" WHO guidelines. However, gaps remain in the VL cascade of care, including missed tests, implementation of enhanced adherence counselling, delays in following up patients with detectable viraemia and delayed switching to second-line treatment after virologic failure is confirmed [9]. Optimizing the VL cascade and expanding coverage requires addressing challenges to VL implementation in resourcepoor settings which include poor adherence to WHO and national guidelines on VL testing, lack of awareness of the benefits of VL testing by clinicians and patients, weak health and laboratory systems, low levels of staff training, poor quality assurance leading to sample rejections, high costs for VL consumables, reagents and tests, and lack of civil society mobilization to improve access to VL testing [12]. Inadequate training, lack of knowledge, poor record-keeping, and difficulties with transportation of samples to the central laboratory are barriers to scale-up of VL testing and may have contributed to the non-uptake of VL testing we observed [17, 26]. Strategies to improve uptake of VL testing including point-of-care testing [28], identifying VL focal persons to flag those in need of VL testing, enhanced adherence counseling, patient education, and demand creation for VL testing, are needed to improve coverage of viral suppression and HIV epidemic control in resource-limited settings [29]. A VL champion program increased testing uptake in South Africa from 64 to $90 \%$ after 6 months [24]. Quality improvement tools focused on providers and patients improved VL testing uptake in Malawi [26]. Implementation of these strategies may help attain the "third 95" target in Uganda, where VL coverage increased from $10 \%$ in 2014 to $85 \%$ in 2019 but is still below the target of universal access [26, 27].

We observed significantly higher non-uptake of VL testing among younger PWH. Younger people below age 25 are less likely to receive a VL test relative to those $>25$ years [27]. Delivery of HIV services to young people, particularly young men, is challenging even in the setting of well conducted test-and-treat trials [30]. Younger age is associated with lower rates of viral suppression and virologic rebound and higher loss to follow-up [31-33]. However, studies in Myanmar and Zimbabwe did not find associations between age and VL testing uptake [34, 35].

We found that PWH who had been on ART for $\leq 2$ years had a two-fold higher odds of non-uptake of VL testing than those who had been on ART for more than 4 years. Relatively similar findings were found in a retrospective cohort study in Mozambique in which duration of ART $\leq 12$ months was significantly associated with non-uptake of VL testing [13]. Shorter duration on ART is also associated with higher odds of viral nonsuppression [12]. To identify patients with unsuppressed VL in need of adherence interventions and at risk of virologic failure, providers should prioritize VL testing after 6 months of ART [36] in accordance with WHO guidelines. Timely targeted adherence counseling decreases the risk of treatment failure and drug resistance, negating the need for more expensive second-line or third-line therapy [37].

The strengths of our cross-sectional study include proportionate allocation methods to randomly select $\mathrm{PWH}$ from all eight HIV clinics in Gomba district, and realworld evaluation of VL non-uptake in a rural setting during scale up of VL testing in Uganda. Our study has limitations. Our cross-sectional design did not permit analysis of time-varying covariates, which may influence trends in VL uptake. We retrospectively analyzed routinely collected program data and there were missing data on marital status (10\%). Nevertheless, this did not influence study findings. We did not analyse data on education, occupation, household income, and distance to health facilities because they are not routinely collected in public health facilities. Finally, our results are not generalizable beyond rural settings. 
In conclusion, we found non-uptake of VL testing in one-third of PWH in rural Uganda. Non-uptake was associated with younger age, shorter duration of ART, and receipt of HIV care at a higher-level primary care facility. Strategies to improve uptake of VL testing, including VL focal persons to flag those in need of VL testing, enhanced adherence counseling, patient education and demand creation for VL testing, are needed to improve coverage of viral suppression and HIV epidemic control in resource-limited settings.

\section{Abbreviations}

ART: Anti-Retroviral Treatment; HIV: Human immunodeficiency virus; UNAIDS: Joint United Nations Program on HIV/AIDS; PWH: People with HIV; VL: Viral Load; WHO: World Health Organization

\section{Acknowledgments}

The authors thank the District Health Officer and other Gomba district personnel whose contributions made this work possible.

\section{Authors' contributions}

RN designed the study and wrote the first draft along with AM. RM, and MRK collected the data and performed the statistical analyses. GK, NM, CNK, PA, JM, and HK participated in the study conception and interpretation of results. DH, FMK, and CA supervised all stages of study implementation. All the authors read and approved the final manuscript.

\section{Funding}

This study was solely funded by RN. AM was supported by the National Institutes of Health (grants K43 TW010695 and R34 MH121084). The content is entirely the responsibility of the authors and does not necessarily represent the official views of the National Institutes of Health or Gomba district administration.

\section{Availability of data and materials}

The dataset used and analyzed during this study is available from the corresponding author on reasonable request.

\section{Ethics approval and consent to participate}

Approval to conduct this retrospective chart review was obtained from the University of Liverpool Board of Ethics (H00057734), Mildmay Uganda Research Ethics Committee (0208-2018) and Uganda National Council for Science and Technology (HS255ES). Administrative clearance was obtained from the District Health Officer of Gomba district.

\section{Consent for publication}

Not applicable.

\section{Competing interests}

The authors declare that they have no competing interests.

\section{Author details}

${ }^{1}$ Makerere University-Johns Hopkins University (MU-JHU) Care LTD, Kampala, Uganda. ${ }^{2}$ Baylor College of Medicine Children's Foundation, Kampala, Uganda. ${ }^{3}$ School of Public Health, College of Health Sciences, Makerere University, Kampala, Uganda. ${ }^{4}$ MRC/UVRI \& LSHTM Uganda Research Unit, Entebbe, Uganda. ${ }^{5}$ School of Social Sciences, College of Humanities and Social Sciences, Makerere University Kampala, Kampala, Uganda. ${ }^{6}$ Department of Public Health and Preventive Medicine, School of Medicine, University of Liverpool, Liverpool, UK. ${ }^{7}$ Infectious Diseases Institute, College of Health Sciences, Makerere University, Kampala, Uganda.

Received: 24 June 2020 Accepted: 30 September 2020 Published online: 06 October 2020

\section{References}

1. UNAIDS. UNAIDS Data 2019. Geneva: Joint United Nations Programme on HIV/AIDS; 2019 ..
2. Lecher S, Williams J, Fonjungo PN, Kim AA, Ellenberger D, Zhang G, Toure CA, Agolory S, Appiah-Pippim G, Beard S, et al. Progress with scale-up of HIV viral load monitoring - seven sub-Saharan African countries, January 2015-June 2016. MMWR Morb Mortal Wkly Rep. 2016;65(47):1332-5.

3. UNAIDS: Understanding Fast-Track: Accelerating Action to End the AIDS Epidemic by 2030. Author Geneva; 2015.

4. UNAIDS. Uganda Country Factsheet. Geneva: UNAIDS; 2019. Available from: https://www.unaids.org/en/regionscountries/countries/uganda. [cited 2019 August 19].

5. Cohen MS, Smith MK, Muessig KE, Hallett TB, Powers KA, Kashuba AD. Antiretroviral treatment of HIV-1 prevents transmission of HIV-1: where do we go from here? Lancet. 2013;382(9903):1515-24.

6. INSIGHT START Study Group, Lundgren JD, Babiker AG, Gordin F, Emery S, Grund B, Sharma S, Avihingsanon A, Cooper DA, Fatkenheuer G, et al. Initiation of antiretroviral therapy in early asymptomatic HIV infection. N Engl J Med. 2015;373(9):795-807.

7. Mujugira A, Celum C, Coombs RW, Campbell JD, Ndase P, Ronald A, Were E, Bukusi EA, Mugo N, Kiarie J, et al. HIV transmission risk persists during the first 6 months of antiretroviral therapy. J Acquir Immune Defic Syndr. 2016; 72(5):579-84.

8. WHO. Consolidated guidelines on the use of antiretroviral drugs for treating and preventing HIV infection: recommendations for a public health approach. Geneva: World Health Organization; 2016.

9. Glass TR, Motaboli L, Nsakala B, Lerotholi M, Vanobberghen F, Amstutz A, Lejone TI, Muhairwe J, Klimkait T, Labhardt ND. The viral load monitoring cascade in a resource-limited setting: a prospective multicentre cohort study after introduction of routine viral load monitoring in rural Lesotho. PLoS One. 2019;14(8):e0220337.

10. UAC. The Uganda HIV and AIDS Country Progress Report July 2015-June 2016. Kampala: Uganda AIDS Commission; 2016.

11. Ministry of Health. Uganda Population-Based HIV Impact Assessment (UPHIA) 2016-2017. Kampala: Ministry of Health Uganda; 2017.

12. Roberts T, Cohn J, Bonner K, Hargreaves S. Scale-up of routine viral load testing in resource-poor settings: current and future implementation challenges. Clin Infect Dis. 2016;62(8):1043-8.

13. Swannet S, Decroo T, de Castro S, Rose C, Giuliani R, Molfino L, Torrens AW, Macueia W, Perry S, Reid T. Journey towards universal viral load monitoring in Maputo, Mozambique: many gaps, but encouraging signs. Int Health. 2017;9(4):206-14.

14. Kerschberger B, Hilderbrand K, Boulle AM, Coetzee D, Goemaere E, De Azevedo V, Van Cutsem G. The effect of complete integration of HIV and TB services on time to initiation of antiretroviral therapy: a before-after study. PLoS One. 2012;7(10):e46988.

15. Rutstein SE, Golin CE, Wheeler SB, Kamwendo D, Hosseinipour MC, Weinberger M, Miller WC, Biddle AK, Soko A, Mkandawire M, et al. On the front line of HIV virological monitoring: barriers and facilitators from a provider perspective in resource-limited settings. AIDS Care. 2016;28(1):1-10

16. Uganda Bureau of Statistics. The national population, and housing census 2014-main report. Kampala; Uganda Bureau of Statistics; 2016.

17. District Health Officer Gomba: Annual District health sector performance report. 2018.

18. Orem JN, Zikusooka CM. Health financing reform in Uganda: how equitable is the proposed National Health Insurance scheme? Int J Equity Health. 2010;9(1):23.

19. Ministry of Health. Consolidated Guidelines on the Prevention and Treatment of HIV in Uganda. Kampala: Ministry of Health; 2018.

20. Kish L. Survey sampling; 1965.

21. Rao T. Optimum allocation of sample size and prior distributions: a review. Int Stat Rev. 1977:173-9.

22. Ministry of Health: National HIV Testing Services Policy and Implementation Guidelines. 2016.

23. Hosmer DW, Lemesbow S. Goodness of fit tests for the multiple logistic regression model. Commun Statist-Theory Methods. 1980;9(10):1043-69.

24. Sunpath $H$, Hatlen T, Naidu K, Adams R, Moosa M, Marconi V, Murphy R, Gandhi R, Pillay S, Siedner M. Targeting the third ' 90 ': introducing the viral load champion. Public Health Action. 2018;8(4):225-31.

25. Awungafac G, Amin ET, Fualefac A, Takah NF, Agyingi LA, Nwobegahay J, Ondoa P, Njukeng PA. Viral load testing and the use of test results for clinical decision making for HIV treatment in Cameroon: an insight into the clinic-laboratory interface. PLoS One. 2018;13(6):e0198686.

26. Lecher S, Ellenberger D, Kim AA, Fonjungo PN, Agolory S, Borget MY, Broyles L, Carmona S, Chipungu G, De KC, et al. Scale-up of HIV viral load 
monitoring--seven sub-Saharan African countries. MMWR Morb Mortal Wkly Rep. 2015;64(46):1287-90.

27. PEPFAR. PEPFAR Uganda country operational plan (COP). Washington, D.C: State Department; 2019.

28. Drain PK, Dorward J, Violette LR, Quame-Amaglo J, Thomas KK, Samsunder N, Ngobese H, Mlisana K, Moodley P, Donnell D, et al. Point-of-care HIV viral load testing combined with task shifting to improve treatment outcomes (STREAM): findings from an open-label, non-inferiority, randomised controlled trial. Lancet HIV. 2020;7(4):e229-37.

29. MSF. Making viral load routine. In: Successes and challenges in the implementation of routine HIV viral load monitoring. vol. Part 1: Medecins Sans Frontieres; 2016.

30. Petersen M, Balzer L, Kwarsiima D, Sang N, Chamie G, Ayieko J, Kabami J, Owaraganise A, Liegler T, Mwangwa F, et al. Association of implementation of a universal testing and treatment intervention with HIV diagnosis, receipt of antiretroviral therapy, and viral suppression in East Africa. JAMA. 2017; 317(21):2196-206.

31. Mujugira A, Celum C, Tappero JW, Ronald A, Mugo N, Baeten JM. Younger age predicts failure to achieve viral suppression and Virologic rebound among HIV-1-infected persons in Serodiscordant partnerships. AIDS Res Hum Retrovir. 2016;32(2):148-54.

32. Etoori $\mathrm{D}$, Ciglenecki I, Ndlangamandla M, Edwards CG, Jobanputra K Pasipamire M, Maphalala G, Yang C, Zabsonre I, Kabore SM, et al. Successes and challenges in optimizing the viral load cascade to improve antiretroviral therapy adherence and rationalize second-line switches in Swaziland. J Int AIDS Soc. 2018;21(10):e25194.

33. Mutevedzi PC, Lessells RJ, Rodger AJ, Newell ML. Association of age with mortality and virological and immunological response to antiretroviral therapy in rural south African adults. PLoS One. 2011;6(7):e21795.

34. Thinn KK, Thekkur P, Kyaw NTT, Aye NS, Zaw TM, Soan P, Hone S, Oo HN. Uptake of routine viral load testing among people living with HIV and its implementation challenges in Yangon region of Myanmar: a mixedmethods study. BMJ Open. 2019;9(12):e032678.

35. Nyakura J, Shewade HD, Ade S, Mushavi A, Mukungunugwa SH, Chimwaza A, Owiti P, Senkoro M, Mugurungi O. Viral load testing among women on 'option B+' in Mazowe, Zimbabwe: how well are we doing? PLoS One. 2019;14(12):e0225476.

36. Joseph Davey D, Abrahams Z, Feinberg M, Prins M, Serrao C, Medeossi B, Darkoh E. Factors associated with recent unsuppressed viral load in HIV-1infected patients in care on first-line antiretroviral therapy in South Africa. Int J STD AIDS. 2018;29(6):603-10.

37. Kerschberger B, Boulle AM, Kranzer K, Hilderbrand K, Schomaker M, Coetzee $D$, Goemaere $E$, Van Cutsem $G$. Superior virologic and treatment outcomes when viral load is measured at 3 months compared to 6 months on antiretroviral therapy. J Int AIDS Soc. 2015;18(1):20092.

\section{Publisher's Note}

Springer Nature remains neutral with regard to jurisdictional claims in published maps and institutional affiliations.

Ready to submit your research? Choose BMC and benefit from:

- fast, convenient online submission

- thorough peer review by experienced researchers in your field

- rapid publication on acceptance

- support for research data, including large and complex data types

- gold Open Access which fosters wider collaboration and increased citations

- maximum visibility for your research: over $100 \mathrm{M}$ website views per year

At $\mathrm{BMC}$, research is always in progress.

Learn more biomedcentral.com/submissions 